\title{
Inflammatory Bowel Disease Cohort Studies in Korea: Present and Future
}

\author{
Jung Won Lee ${ }^{1}$, Jong Pil Im ${ }^{1}$, Jae Hee Cheon ${ }^{2}$, You Sun Kim³ ${ }^{3}$ Joo Sung Kim¹, Dong Soo Han ${ }^{4}$ \\ Department of Internal Medicine and Liver Research Institute, Seoul National University College of Medicinel, Department of Internal Medicine \\ and Institute of Gastroenterology, Yonsei University College of Medicine, Department of Internal Medicine, Inje University College of Medicine, \\ Seoul, Department of Internal Medicine, Hanyang University College of Medicine 4 , Guri, Korea
}

Inflammatory bowel disease (IBD) is defined as a chronic and relapsing inflammatory disorder of the intestine. Intestinal inflammation in IBD has been proposed to be attributable to the interplay between microbial, genetic, environmental, and immunological factors. The incidence and prevalence rates of IBD are rapidly increasing apparently in other parts of the world, with dramatic increases especially in East Asia. Generally, cohort studies are useful for estimating the incidence, prevalence, natural course, prognosis, and risk factors of diseases. In particular, cohort studies performed in Western countries have well described the prevalence, risk factors, and natural course of IBD and investigated its genetic pathophysiology. However, the outcomes of IBD cohort studies performed in Korea are not as persuasive as those of Western studies because of the relatively low prevalence of IBD and short follow-up periods of the cohorts in Korea. Despite this critical limitation, members of the Korean Association for the Study of Intestinal Diseases have demonstrated outstanding results. Some unique features of IBD patients in Korea are well demonstrated, such as thiopurine-induced leukopenia or risks of opportunistic tuberculosis infection in patients receiving tumor necrosis factor- $\alpha$ inhibitors. In this review, the present authors summarized the key points of the results of the cohort studies performed in Korea and explored future perspectives. (Intest Res 2015;13:213-218)

Key Words: Inflammatory bowel diseases; Cohort studies; Korea

\section{INTRODUCTION}

Inflammatory bowel disease (IBD), CD, and UC are chronic relapsing inflammatory disorders of the intestine with unknown causes. IBD develops upon the interaction between genetic predisposition leading to immunological abnormalities, environment, and microbiome. None of these factors alone are sufficient to induce disease development. ${ }^{1}$

The occurrence of IBD is increasing in North America, Europe, and worldwide. ${ }^{2,3}$ The incidence and prevalence of

Received April 23, 2015. Revised April 26, 2015. Accepted April 30, 2015. Correspondence to Jong Pil Im, Department of Internal Medicine and Liver Research Institute, Seoul National University Hospital, 101 Daehak-ro, Jongno-gu, Seoul 110-744, Korea. Tel: +82-2-2072-0638, Fax: +82-2-7629662,E-mail: jpim0911@snu.ac.kr

Financial support: This work was supported by the research program funded by the Korea Centers for Disease Control and Prevention [2013E63004-01]. Conflict of interest: None.
IBD in Asia remain low compared with those in Western countries, but a rising trend has been recognized in recent years, providing strong evidence in support of the effect of the environment. ${ }^{4-7}$ The characteristics of Western and Asian patients with IBD differ in epidemiology, phenotype, clinical course, and genetic susceptibility. ${ }^{4,8-10}$ Korea has a relatively small land area and an ethnically homogeneous population. Since the 1960s, when the first IBD cases in Korea were reported, the incidence and prevalence of IBD in Korea has been lower than that in Western countries but have demonstrated rapidly increasing trends. Several distinct characteristics of patients with IBD in Korea have been reported, such as thiopurine-induced leukopenia and the risk of opportunistic tuberculosis (TB) infection in patients administered tumor necrosis factor- $\alpha$ (TNF- $\alpha$ ) inhibitor. ${ }^{11-13}$ These distinct characteristics can provide important clues about etiology and pathophysiology, and comprehensive population-based

\footnotetext{
๑ Copyright 2015. Korean Association for the Study of Intestinal Diseases. All rights reserved.

This is an Open Access article distributed under the terms of the Creative Commons Attribution Non-Commercial License (http://creativecommons.org/licenses/by-nc/4.0)

which permits unrestricted non-commercial use, distribution, and reproduction in any medium, provided the original work is properly cited.
} 
studies are required to clarify these differences. ${ }^{6}$

A cohort study is generally useful for estimating the incidence, prevalence, disease course, prognosis, and other clinically distinct features. Cohort studies conducted in Western Europe and America have described the IBD clinical course and identified its pathophysiology. Some unique features of patients with IBD in Korea have been well demonstrated by cohort studies in Korea, especially by members of the Korean Association for the Study of Intestinal Diseases (KASID). However, most of the currently available data came from single-center and a few multi-center studies those were primarily retrospective in design. ${ }^{6}$ Potential problems with the retrospective cohort approach include selection and misclassification bias. Moreover, there have been no welldesigned prospective cohort studies in Korea. From this background, here we prepare the key findings of cohort studies of patients with IBD in Korea to date and explore future potential IBD cohort studies in Korea.

\section{PAST AND PRESENT}

The first systematic population-based cohort study was performed in the Songpa-Kangdong district of Seoul in 1986. ${ }^{14}$ According to a recent update on the descriptive epidemiology of the Songpa-Kangdong district, the mean annual incidences of CD and UC increased significantly from 0.05 and 0.34 per 100,000 inhabitants, respectively, in 1986-1990 to 1.34 and 3.08 per 100,000 inhabitants, respectively, in 2001-2005. During this 20-year period, the ratio of the incidences of UC to CD was decreasing from 6.8 in 1986-1990 to 2.3 in 2001-2005, indicating that CD demonstrated a trend of a more accelerated incidence compared than UC in Korea. ${ }^{15}$ The clinical features of UC at diagnosis are reportedly similar in Koreans and Westerners. For example, there are no differences in disease extent and severity at diagnosis or in the male-to-female ratio of patients with UC between Korea and Western countries. The cumulative risk of proximal extension in patients with proctitis or left-sided colitis was $33.0 \%$ after 5 years and $44.5 \%$ after 10 years. The cumulative probability of colectomy was $2.0 \%$ after 1 year, $2.8 \%$ after 3 years, and $3.3 \%$ after $5-15$ years. The cumulative survival rates after 1, 5, and 10 years were $100 \%, 99.4 \%$, and $97.4 \%$, respectively. Korean patients with UC look like to have a milder course that results in a reduced risk of colectomy compared to the Western population. ${ }^{16}$ Approximately $1.1 \%$ of patients with UC are diagnosed with any UC-related symptoms. Most of these asymptomatic UC patients become symptomatic during follow-up but appeared to have better prognosis than symptomatic UC patients. ${ }^{17}$
A male predominance in the prevalence of CD was observed in the Korean population, a finding similar to those reported in recent studies from China and Japan, ${ }^{18}$ while the incidence of $\mathrm{CD}$ in females is predominant or equal to that in males in Western countries. ${ }^{4,19}$ Patients with CD may have a similar clinical course to Westerners, especially regarding the intestinal resection rate. The surgery rate has decreased as time passes, which was related to the early administration of azathioprine/6-mercaptopurine (AZA/6-MP). ${ }^{20} \mathrm{How}$ ever, Korean patients with CD are more likely than Western patients to have jejunal involvement. Jejunal involvement is a factor of poor prognosis in both Korean and Western patients with $\mathrm{CD}^{21}$ Moreover, perianal fistulas seemed more common in Korean patients than in Westerners. ${ }^{22}$

The early administration of biological agents showed improved outcomes of IBD; consequently, increasing numbers of patients with IBD are expected to receive TNF- $\alpha$ inhibitor therapy. ${ }^{23,24}$ One of the most feared side effects is an increased risk of opportunistic TB infection, especially in an IBD population from a TB endemic area. ${ }^{25,26}$ The KASID conductive retrospective cohort study aimed to define the risk of TB in a large cohort of 873 IBD patients using TNF- $\alpha$ inhibitors compared with the matched general population and identify the detailed clinical characteristics of and risk factors for newly developed TB in patients with IBD. A total of 25 newly developed TB cases-21 (84\%) pulmonary and $4(16 \%)$ extra-pulmonary infections-were identified in the cohort. The adjusted standardized incidence ratio (SIR) of TB was 41.7 (95\% CI, 25.3-58.0), but the outcomes of anti-TB treatment were primarily favorable. ${ }^{27}$

The risks and characteristics of colorectal cancer (CRC) in IBD were also assessed. ${ }^{28}$ The population-based nationwide study was conducted to investigate the incidence of CRC in patients with UC. The KASID reviewed 7,061 cases of UC that occurred between 1970 and 2005 and found a total of 26 cases of CRC. They reported that the cumulative risk of UC-associated CRC was $0.7 \%$ for patients that had UC for 10 years, $7.9 \%$ for 20 years, and $33.2 \%$ for 30 years. The mean duration of UC before the development of CRC was 11.5 years. ${ }^{29}$ Another study from a well-defined hospital-based cohort was recently published. A total of 5,212 patients with IBD (2,414 with CD and 2,798 with UC) were followed up for 39,951 person-years, thus a mean follow-up duration of 7.7 years. Thirty patients ( 12 with CD and 18 with UC) developed CRC. The SIR of CRC was 6.0 (95\% CI, 3.10-10.48) for CD and 1.68 (95\% CI, 1.00-2.66) for UC. The SIR of CRC in Korean patients with UC may be similar to that in Western patients with UC. In contrast to Western patients with CD, the most common site of CRC in Korean patients with CD 
is the low rectum, probably in accordance with the higher prevalence of perianal fistulas in Korean patients with CD. ${ }^{30}$

Thiopurine-associated leukopenia could be a life-threatening side effect of AZA/6-MP in IBD treatment, and Korean patients seem to experience myelotoxicity more frequently (31-56\%) than patients of European descent. ${ }^{11}$ Although thiopurine methyltransferase (TPMT) genotypes and activity could not clarify the thiopurine-induced leukopenia completely, it was proposed that TPMT genotypes and activity measurements before AZA/6-MP administration might help to predict the development of leukopenia. ${ }^{31}$ Recently, using the sample from cohort of $978 \mathrm{CD}$ patients treated with thiopurine, a nonsynonymous single nucleotide protein (SNP) in NUDT15 (encoding p.Arg139Cys) was strongly associated with thiopurine-induced early leukopenia in both Korean and United States patients with IBD. ${ }^{32}$ These findings explain, in part, the higher prevalence of thiopurine-associated leukopenia in Asians despite the lower prevalence of TPMT mutations. These results highlight genetic or biomarker studies require blood or tissue specimens from a large number of patients with accurate, matched clinical data.

In addition to leukopenia, growing evidence supports the existence of a correlation between thiopurine treatment and the increased risk of developing lymphoma. Patients with lymphoma and IBD from three tertiary referral centers in Korea were analyzed. Seven cases of lymphoma $(0.1 \%)$ were identified in 6,585 patients with IBD. Three patients had UC and 4 had CD. Non-Hodgkin lymphoma was diagnosed in 5 patients (71.4\%) and Hodgkin disease in 2 patients (28.6\%). The SIR of lymphoma was 2.03 (95\% CI, 0.81-4.18) in the entire IBD patients and the SIR of lymphoma in patients with CD was 9.31 (95\% CI, 1.13-33.62). The SIR of lymphoma in patients who were exposed to thiopurines was 5.93 (95\% CI, 1.61-15.18). The risk of lymphoma in patients with CD seems to be increased and thiopurine may be related with the risk of lymphoma in Korean patients with IBD. ${ }^{33}$
Since genome-wide association studies (GWAS) have been introduced in an effort to identify the etiological factors of IBD, ${ }^{34}$ many genetic polymorphisms related to an increased risk of IBD have been identified based on an established IBD cohort. ${ }^{35}$ TNFSF15 is a proven susceptibility gene for CD. The associations among five TNFSF15 SNPs and various clinical parameters were investigated using a total of 906 patients with CD and TNFSF15 genotype data with clinical information. In Korean patients with CD, nonrisk allele homozygotes of the TNFSF15 SNPs rs6478108 and rs4574921 are independent genetic predictive factors for the development of strictures/non-perianal penetrating complications and perianal fistula. ${ }^{36}$ GWAS was performed in the Korean population comprising a total of 2,311 patients with CD, 2,442 controls derived from the IBD Clinic of Asan Medical Center, and 792 from the Korea Research Network for Crohn's Disease. In this study, three new susceptibility loci demonstrated genome-wide significance: rs6856616 at 4 p14 (OR, 1.43; combined $\left.P=3.60 \times 10^{-14}\right)$, rs11195128 at $10 \mathrm{q} 25$ (OR, 1.42; combined $P=1.55 \times 10^{-10}$ ), and rs11235667 at $1 \mathrm{lq} 13\left(\mathrm{OR}, 1.46\right.$; combined $\left.P=7.15 \times 10^{-9}\right){ }^{37}$

\section{FUTURE}

Attempts to comprehensively understand the epidemiologic, clinical, and genetic characteristics of Korean patients with CD first started in 2008. Won Ho Kim, President of the KASID at that time, earned a national grant entitled the "Research Network for Crohn's Disease." During the study period, active translational research was performed using clinical data from about 2,000 blood samples of 1,316 Korean patients with $\mathrm{CD}$. The study team also established the Korean diagnostic and therapeutic guidelines for CD with the help of the IBD Study Group of KASID that published the diagnostic guidelines for intestinal $\mathrm{TB}$, a disease often misdiagnosed as CD. ${ }^{6}$ After the Research Network for Crohn's

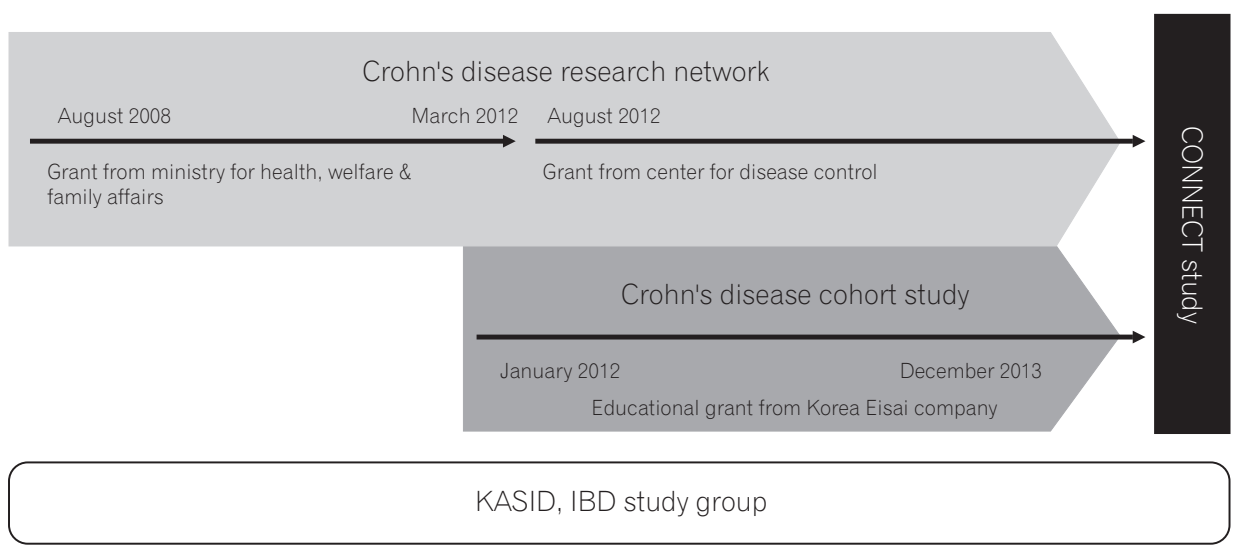

Fig. 1. The progress of three studies of the Crohn's Disease Clinical Network and Cohort (CONNECT) study. KASID, Korean Association for the Study of Intestinal Diseases. ${ }^{6}$ 
Disease study, a subsequent study named "Establishment of Crohn's disease in Korea and characterization of clinical features with long-term follow-up" was conducted from January 2012 to December 2013 led by Dong Soo Han. Retrospective clinical data before 2009 and prospective data for patients enrolled during and after 2009 were collected using a novel web-based well-recorded, high-quality electronic case report form system. Data for 1,388 retrospective patients and 890 prospective patients including 635 blood specimens were collected and analyzed.

In 2012, Korea Centers for Disease Control and Prevention solicited grant applications for a 6-month demonstration project aimed at establishing a CD network. The KASID obtained this grant, and in 2012, a 3-year national grant from the Korea Centers for Disease Control and Prevention. The new study funded by these grants was named "Establishment and Management of Crohn's Disease Research Network". Recently, the latter two studies discussed above were integrated into a single study referred to as the "Crohn's Disease Clinical Network and Cohort" (CONNECT) study led by Joo Sung Kim (Fig. 1).

A total of 34 institutions are now participating in this study, and more than 2,000 blood specimens have been collected to date. More than 20 studies using a retrospective cohort of 1,382 patients are underway and some results were presented at the KASID, Asian Organization for Crohn's and Colitis, and European Crohn's and Colitis Organization meetings. The baseline characteristics of patients with a follow-up period $\geq 6$ months are shown in Table 1 . Men comprised $69.5 \%$ and the median age at diagnosis was 24 years. A total of $2.1 \%$ of patients had a family history of IBD. According to the Montreal classification, ileocolonic location (L3) was the most common, followed by the ileal (L1) and colonic (L3) locations. In 1,016 (79.5\%) patients, the disease behavior at diagnosis was inflammatory. Of the patients, 560 (43.8\%) and $144(11.3 \%)$ had perianal disease and stenosis at diagnosis, respectively.

The first result led by Dong Il Park was recently published about long-term clinical outcomes of Korean patients with $\mathrm{CD}$ in urban versus rural environments. Although numerous studies have reported a positive correlation between urban environment and CD development, no differences were found in terms of disease presentation and natural history between Korean urban and rural populations except for a higher rate of surgery in the urban population of patients recently diagnosed with $\mathrm{CD}^{38}$ You Sun Kim et al. conducted the study to determine the incidence of free perforation, the clinical characteristics of patients with CD and free perfora- tion, and the risk factors associated with free perforation in patients with CD in Korea. Eighty-eight (6.4\%) of the 1,382 patients had free perforation, and multivariate analysis revealed that free perforation was significantly associated with age $\geq 30$ years at diagnosis (OR, 2.082; $P=0.002$ ) and bowel stricture (OR, 1.982; $P=0.004))^{39}$ These retrospective databased studies will be the background for future prospectively enrolled cohort studies to investigate the clinical course and pathophysiology of Korean patients with CD. As of March 2015, 1,302 patients diagnosed with CD after 2009 with 850

Table 1. Baseline Characteristics of Retrospective Cohort Study With Follow-Up Period of 6 Months or More

\begin{tabular}{|c|c|}
\hline Characteristics & CD cohort $(n=1,278)$ \\
\hline \multicolumn{2}{|c|}{ Baseline characteristics at diagnosis } \\
\hline \multicolumn{2}{|l|}{ Demographic data } \\
\hline Age, yr, median (range) & $24.0(7-87)$ \\
\hline Male gender & $888(69.5)$ \\
\hline $\mathrm{BMI}, \mathrm{kg} / \mathrm{m}^{2}$, median (range) & $19.0(12-41)$ \\
\hline Family history of IBD & $27(2.1)$ \\
\hline Presence of granuloma & $412(32.2)$ \\
\hline \multicolumn{2}{|l|}{ Location (involvement) } \\
\hline Jejunum & $200(15.6)$ \\
\hline lleum & $1,003(78.5)$ \\
\hline Colorectal & $941(73.6)$ \\
\hline Upper GI tract & $91(7.1)$ \\
\hline \multicolumn{2}{|l|}{ Montreal classification } \\
\hline \multicolumn{2}{|l|}{ Age at diagnosis } \\
\hline$<16$ yr $(\mathrm{A} 1)$ & $121(9.5)$ \\
\hline $17-40$ yr (A2) & $972(76.1)$ \\
\hline$>40$ yr (A3) & $178(13.9)$ \\
\hline \multicolumn{2}{|l|}{ Location } \\
\hline lleal (L1) & $291(22.8)$ \\
\hline Colonic (L2) & $229(17.9)$ \\
\hline Ileocolonic (L3) & $712(55.7)$ \\
\hline Isolated upper disease (L4) & $15(1.2)$ \\
\hline \multicolumn{2}{|l|}{ Behavior } \\
\hline Inflammatory (B1) & $1,016(79.5)$ \\
\hline Stricturing (B2) & $120(9.4)$ \\
\hline Penetrating (B3) & $143(11.2)$ \\
\hline Perianal lesions & $560(43.8)$ \\
\hline Stenosis & $144(11.3)$ \\
\hline
\end{tabular}

Values are presented as $n(\%)$.

$\mathrm{Gl}$, gastrointestinal. 
serum samples are registered and prospectively followed-up in the national CONNECT study. ${ }^{6}$ We regularly audit the data and send a newsletter to the investigators to remind them of the importance of accurate and complete data acquisition. Investigators from the CONNECT study anticipate that the typical clinical characteristics and genetic causes of CD in Korean patients will be elucidated.

Despite the established nationwide CD cohort study in Korea, there has been little nationwide systematic data on epidemiology, clinical characteristics, and genetics of Korean patients with UC. There is an urgent need for the nationwide collection of well-defined clinical characteristics of Korean patients with UC and their blood samples. Hyo-Jong Kim, as coordinating investigator, and the other associated KASID members initiated the first nationwide "Cohort study of newly diagnosed moderate to severe ulcerative colitis in Korea" (MOSAIK). This is a multicenter, nationwide, prospective, 5-year follow-up longitudinal, disease-oriented, and hospitalbased cohort study. Patients newly diagnosed with moderate to severe UC in tertiary referral centers are to be included in this study. The aim of the MOSAIK study is to establish a UC research cohort for the investigation of epidemiology, clinical characteristics, and genetics of Korean patients with UC by collecting well-defined clinical and follow-up characteristics of Korean patients with UC and their blood samples with a goal of recruiting 400 patients. As of April 2015, >100 patients diagnosed with UC from 27 hospitals are registered. The MOSAIK cohort will be closed when the last patient completes the last scheduled 5-year follow-up visit. The clinical characteristics and genetic causes of Korean patients with UC could be elucidated through the MOSAIK cohort study results.

\section{CONCLUSIONS}

It is widely accepted that a cohort study with a prospective design would be a very appropriate for epidemiologic study. No well-designed nationwide cohort study of IBD has been published in Korea to date. However, recent long-term follow-up results from the IBD cohort study might help clinicians and researchers to make more clear decisions. These findings, together with the distinct demographic and phenotypic characteristics of Korean patients with IBD, may provide clues to the etiology of these diseases as well as useful information for health care policy. Even though the initiation of IBD cohort studies in Korea was quite behind compared to Western studies, there are some clinical and genotypic characteristics unique to Korean patients with IBD like thio- purine-induced side effects and opportunistic TB infections. These unique features could provide important implications about the etiology and pathophysiology of IBD through a comprehensive population-based study. Moreover, studies regarding biosamples might strengthen the impact of the cohort study.

In an IBD cohort study, the typical clinical characteristics and genetic causes of Korean patients with IBD could be elucidated, which will be a basis for further research into the clinical and genetic characteristics of IBD in Korea. Further diagnosis and treatment guidelines and new therapeutic agents for Korean patients with IBD will be developed based on these cohort findings.

\section{REFERENCES}

1. Maloy KJ, Powrie F. Intestinal homeostasis and its breakdown in inflammatory bowel disease. Nature 2011;474:298-306.

2. Burisch J, Pedersen N, Cukovic-Cavka S, et al. Environmental factors in a population-based inception cohort of inflammatory bowel disease patients in Europe-an ECCO-EpiCom study. J Crohns Colitis 2014;8:607-616.

3. Molodecky NA, Soon IS, Rabi DM, et al. Increasing incidence and prevalence of the inflammatory bowel diseases with time, based on systematic review. Gastroenterology 2012;142:46-54.

4. Kim ES, Kim WH. Inflammatory bowel disease in Korea: epidemiological, genomic, clinical, and therapeutic characteristics. Gut Liver 2010;4:1-14.

5. Lee KM, Lee JM. Crohn's disease in Korea: past, present, and future. Korean J Intern Med 2014;29:558-570.

6. Cheon JH, Kim YS, Ye BD, et al. Crohn's Disease Clinical Network and Cohort (CONNECT) Study: the first step toward nationwide multicenter research of Crohn's disease in Korea. Intest Res 2014;12:173-175.

7. Shin DH, Sinn DH, Kim YH, et al. Increasing incidence of inflammatory bowel disease among young men in Korea between 2003 and 2008. Dig Dis Sci 2011;56:1154-1159.

8. Thia KT, Loftus EV, Jr., Sandborn WJ, Yang SK. An update on the epidemiology of inflammatory bowel disease in Asia. Am J Gastroenterol 2008;103:3167-3182.

9. Park SJ, Kim WH, Cheon JH. Clinical characteristics and treatment of inflammatory bowel disease: a comparison of Eastern and Western perspectives. World J Gastroenterol 2014;20:11525-11537.

10. Ali RA. The positive influences of increasing age at diagnosis of inflammatory bowel disease on disease prognostication in asian perspective. Intest Res 2015;13:4-5. 
11. Kim JH, Cheon JH, Kim WH. The frequency and the course of the adverse effects of azathioprine/6-mercaptopurine treatment in patients with inflammatory bowel disease. Korean J Gastroenterol 2008;51:291-297.

12. Lim SM, Nam CM, Kim YN, et al. The effect of the menstrual cycle on inflammatory bowel disease: a prospective study. Gut Liver 2013;7:51-57.

13. Jung YS, Song CS, Kim ER, et al. Seasonal variation in months of birth and symptom flares in Korean patients with inflammatory bowel disease. Gut Liver 2013;7:661-667.

14. Yang SK, Hong WS, Min YI, et al. Incidence and prevalence of ulcerative colitis in the Songpa-Kangdong District, Seoul, Korea, 1986-1997. J Gastroenterol Hepatol 2000;15:1037-1042.

15. Yang SK, Yun S, Kim JH, et al. Epidemiology of inflammatory bowel disease in the Songpa-Kangdong district, Seoul, Korea, 1986-2005: a KASID study. Inflamm Bowel Dis 2008;14:542549.

16. Park SH, Kim YM, Yang SK, et al. Clinical features and natural history of ulcerative colitis in Korea. Inflamm Bowel Dis 2007;13:278-283.

17. Park SK, Ye BD, Yang SK, et al. Clinical features and course of ulcerative colitis diagnosed in asymptomatic subjects. J Crohns Colitis 2014;8:1254-1260.

18. Yao T, Matsui T, Hiwatashi N. Crohn's disease in Japan: diagnostic criteria and epidemiology. Dis Colon Rectum 2000;43:S85S93.

19. Bernstein CN, Wajda A, Svenson LW, et al. The epidemiology of inflammatory bowel disease in Canada: a population-based study. Am J Gastroenterol 2006;101:1559-1568.

20. Park SH, Yang SK, Park SK, et al. Long-term prognosis of Crohn's disease and its temporal change between 1981 and 2012: a hospital-based cohort study from Korea. Inflamm Bowel Dis 2014;20:488-494.

21. Park SK, Yang SK, Park SH, et al. Long-term prognosis of the jejunal involvement of Crohn's disease. J Clin Gastroenterol 2013;47:400-408.

22. Ye BD, Yang SK, Cho YK, et al. Clinical features and long-term prognosis of Crohn's disease in Korea. Scand J Gastroenterol 2010;45:1178-1185.

23. Park SC, Jeen YT. Current and emerging biologics for ulcerative colitis. Gut Liver 2015;9:18-27.

24. Seo HI, Park DI, Kim TO, et al. The effect of infliximab on patients with ulcerative colitis in Korea. Intest Res 2014;12:214220.

25. Chung KB, Lee EY, Im JP, Han SK, Yim JJ. Clinical characteristics and treatment responses of patients who developed tuberculosis following use of a tumor necrosis factor- $\alpha$ inhibitor. Korean J Intern Med 2013;28:174-179.
26. Shim TS. Diagnosis and treatment of latent tuberculosis infection in patients with inflammatory bowel diseases due to initiation of anti-tumor necrosis factor therapy. Intest Res 2014;12:12-19.

27. Byun JM, Lee CK, Rhee SY, et al. Risks for opportunistic tuberculosis infection in a cohort of 873 patients with inflammatory bowel disease receiving a tumor necrosis factor- $\alpha$ inhibitor. Scand J Gastroenterol 2015;50:312-320.

28. Zhiqin W, Palaniappan S, Raja Ali RA. Inflammatory bowel disease-related colorectal cancer in the Asia-Pacific region: past, present, and future. Intest Res 2014;12:194-204.

29. Kim BJ, Yang SK, Kim JS, et al. Trends of ulcerative colitis-associated colorectal cancer in Korea: A KASID study. J Gastroenterol Hepatol 2009;24:667-671.

30. Lee HS, Park SH, Yang SK, et al. The risk of colorectal cancer in inflammatory bowel disease: a hospital-based cohort study from Korea. Scand J Gastroenterol 2015;50:188-196.

31. Kim JH, Cheon JH, Hong SS, et al. Influences of thiopurine methyltransferase genotype and activity on thiopurine-induced leukopenia in Korean patients with inflammatory bowel disease: a retrospective cohort study. J Clin Gastroenterol 2010;44:e242-e248.

32. Yang SK, Hong M, Baek J, et al. A common missense variant in NUDT15 confers susceptibility to thiopurine-induced leukopenia. Nat Genet 2014;46:1017-1020.

33. Park SK, Ye BD, Lee C, et al. Risk and clinical characteristics of lymphoma in Korean patients with inflammatory bowel diseases: a multicenter study. J Clin Gastroenterol 2015;49:e11-e16.

34. Liu JZ, Anderson CA. Genetic studies of Crohn's disease: past, present and future. Best Pract Res Clin Gastroenterol 2014;28:373-386.

35. Rioux JD, Xavier RJ, Taylor KD, et al. Genome-wide association study identifies new susceptibility loci for Crohn disease and implicates autophagy in disease pathogenesis. Nat Genet 2007;39:596-604.

36. Yang DH, Yang SK, Song K, et al. TNFSF15 is an independent predictor for the development of Crohn's disease-related complications in Koreans. J Crohns Colitis 2014;8:1315-1326.

37. Yang SK, Hong M, Zhao W, et al. Genome-wide association study of Crohn's disease in Koreans revealed three new susceptibility loci and common attributes of genetic susceptibility across ethnic populations. Gut 2014;63:80-87.

38. Jung YS, Park DI, Ye BD, et al. Long-term clinical outcomes of urban versus rural environment in Korean patients with Crohn's disease: results from the CONNECT study. J Crohns Colitis 2015;9:246-251.

39. Doh YS, Kim YS, Bae SI, et al. The clinical characteristics of patients with free perforation in Korean Crohn's disease: results from the CONNECT study. BMC Gastroenterol 2015;15:31. 